\title{
Morfología atípica de Cryptococcus neoformans en tejidos autopsiados
}

(Atypical morphology of Cryptococcus neoformans in post-mortem tissues)

Aida van Gelderen ${ }^{1 *}$. Isabel Borges de Kestelman ${ }^{1}$

Cátedra Micología, Instituto de Microbiología «Luis C. Verna», Facultad de Bioquímica, Química y Farmacia, Universidad Nacional de Tucumán. Ayacucho 491, (4000) San Miguel de Tucumán, R. Argentina.

Tel: $54-381-4247752$

*Autor para correspondencia: micologia@ fbqf.unt.edu.ar

Recibido:29-11-12

Aprobado:10-12-12

Palabras clave: Cryptococcus neoformans, células atípicas, morfología.

Key words: Cryptococcus neoformans, atypical cell, morphology.

\section{RESUMEN}

Cryptococcus neoformans es el agente de la Blastomicosis Europea, Torulosis 0 Cryptococcosis. Causa enfermedad en pacientes inmunocomprometidos. Estos hongos viven en distintos ambientes, penetran a los pulmones por inhalación y por diseminación hematógena, pueden comprometer el cerebro, meninges y otros órganos.

En los tejidos invadidos y en su habitat natural se presenta con igual morfología, como levaduras gemantes globosas a ovales, de 4 a $7 \mu$ de diámetro aunque pueden verse células de 2 a $15 \mu$, rodeadas de una gran cápsula de mucopolisacáridos que duplica o triplica el diámetro de las células. Típicamente son células simples 0 con un brote, en ciertas lesiones pueden encontrarse cadenas cortas de 2 a 3 células. Las levaduras están ampliamente separadas por espacios ocupados por sus cápsulas. El objetivo de este trabajo es informar la morfología atípica de un aislamiento de $C$. neoformans en tejidos de órganos: hígado, bazo, riñón y cerebro obtenidos por autopsia de una persona de sexo masculino de 33 años de edad. La Identificación de C. neoformans fue realizada por sus caracteres morfológicos, cultivos y fenotípicos.
En hígado, bazo y riñón se observaron abundantes levaduras grmantes con gran cápsula. Algunas levaduras estaban unidas a las células madres por una base ancha, lo cual no es característica de la especie. Presentaba marcada asociación entre células formando estructuras caprichosas y muy desarrolladas con escasas formas típicas del hongo y pseudomicelios. En cerebro fue más frecuente la observación de elementos gemantes simples.

\section{ABSTRACT}

C. neoformans is the agent of the Cryptococcosis, European Blastomycosis or Torulosis. This fungus can cause disease in normal individuals and in inmunocompromised patients. Infection follows inhalation, but shows a remarkable propensity to spread hematogenously to the brain and meninges and even other organs. $C$. neoformans is an encapsulated budding yeasts, globular to oval in shape, 4 to $7 \mathrm{im}$ in diameter not including capsules, but cells from 2 to 15 im may occur. The yeasts are surrounded by a large mucopolysaccharide capsule which duplicates or triplicates the cell diameter. Cells are typically single or with one bud, but may be found in short chains 
Morfología atípica de Cryptococcus neoformans en tejidos autopsiados - A van Gelderen et al.

of 2 to 3 cells. The yeasts are clearly separated by spaces occupied by capsules.

The aim of this work is to communicate the atypical morphology presented by a $C$. neoformans strain isolated from liver, spleen, kidney and brain tissue. Tissues were obtained through autopsy of a 33-year-old male patient without accurate cause of death. The identification of $C$. neoformans was made by its morphological, culture and phenotypical characteristics.

In liver, spleen and kidney were observed budding yeast abundant with large capsule and numerous buds. The buds were linked to mather cells by a wide base, which is not characteristic of the species. Showed a strong association between cells, without letting go of the parent cell, were capricious and highly developed structures. Presented few typical forms of the fungus and pseudomycelia rudimentary. In brain was more often observed budding simple elements.

\section{INTRODUCCIÓN}

C. neoformans es el agente de la Criptococosis, también llamada Torulosis o Blastomicosis Europea. La Criptococosis constituye un grave problema de salud pública mundial. Su agente, que vive en distintos tipos de sustratos, penetra a los pulmones por inhalación y se disemina por vía hematógena con tendencia a comprometer al cerebro y meninges y otros órganos. Puede actuar como un hongo patógeno primario, capaz de enfermar a personas aparentemente inmunocompetentes, pero es más frecuente su acción como oportunista asociado a múltiples causas predisponentes, en individuos inmunocomprometidos, especialmente en pacientes con SIDA (1-4).

La Criptococosis se ha incrementado desde la aparición del SIDA, llegando a ocupar el cuarto lugar entre las infecciones acompañantes de esta patología, después de la pneumocistosis, la candidiasis bucofaríngea y la tuberculosis $(4,5)$. En estos pacientes, su incidencia varía en diferentes áreas del planeta: entre el 5-10\% en Europa occidental y los Estados Unidos, y más del 20\% en África Central y el sudeste de Asia. (6). Se estima que ocurren más de 1 millón de casos nuevos de Criptococosis por año en el mundo en pacientes con SIDA, de los cuales mueren más de 650.000 por año (7).

En la actualidad, esta entidad esta constituída por un complejo de especies: el «Cryptococcus neoformans complex»: que abarca 5 serotipos ( $A$, $B, C, D, y A D$ ) divididos sobre las bases de las propiedades inmunológicas de los polisacáridos capsulares $(8,9)$ y esta integrado por 2 especies: $C$. neoformans (anteriormente denominado $C$. neoformans var. neoformans, (serotipos D y AD), la variedad: C. neoformans var. grubii (abarca el serotipo A), ambos con teleomorfo de Filobasidiella neoformans var. neoformans y C. gattii (anteriormente $C$. neoformans var. gattii), (serotipos B y C) con teleomorfo en $F$. neoformans var. bacillispora (8-10).

C. neoformans, y C. gattii presentan diferencias clínicas, morfológicas, bioquímicas, serológicas y epidemiológicas (10-15). Los casos por $C$. neoformans han sido descritos en todo el mundo y el agente fue aislado de diferentes fuentes naturales $(15,16)$, mientras que las infecciones por C. gattii están restringidas a las áreas tropicales y subtropicales. La ecología de $C$. gattii es conocida desde 1990, cuando se estableció su asociación ecológica específica con Eucalyptus. camaldulensis y posteriormente con $E$. tereticornis.. También se lo ha relacionado con otras especies de Eucalyptus: $E$. gomphocephala, E. rudis y E. blakelyi $(12,17,20)$. C. gattii fue, también, aislado de guano de murciélago y de una colmena de avispa Polybia occidentales $(21,22)$. C. neoformans es responsable de la mayoría de los casos de criptococcosis in pacientes inmunocomprometidos, y C. gattii se asocia con infecciones en sujetos con estado inmunológico normal $(13,23)$. En la Argentina la mayoría de casos de criptococosis son a C. neoformans, siendo raros los por $C$. gattii (24).

C. neoformans es un hongo monomórfico, con igual morfología en su vida saprofítica y en su fase parasitaria. Es un hongo unicelular, sus células son esféricas 0 elipsoidales, 0 de forma de limón de 3,5-7,5 × 3-7 $\mu$, con cápsulas gruesas de mucopolisacáridos que pueden llegar a triplicar el diámetro de la levadura y que representan el principal 
Morfología atípica de Cryptococcus neoformans en tejidos autopsiados - A van Gelderen et al.

factor de virulencia del hongo $(1,2,10,25-28)$. Se reproduce por gemación, y los brotes pueden producirse en cualquier lugar de las células (brotación multilateral) (25). No es capaz de formar pseudohifas o hifas, característica importante para su diferenciación con Candida. La brotación de las células es su mecanismo asexuado de reproducción. Los brotes se unen a las células madres por una base angosta $(1,2,10,25)$.

Se han reportado casos atípicos, ocasionales, donde $C$. neoformans mostró alteraciones de forma y de tamaño (hasta 40 a $50 \mu$ de diámetro) en criptococcosis experimental y en casos humanos, en inmunocompetentes e inmunocomprometidos. También, fue informada la formación de cadenas de levaduras brotantes, pseudohifas, estructuras semejantes a tubos germinativos, hifas cortas aberrantes y células altamente 0 pobremente capsuladas (29-36).

Para contribuir al conocimiento de la diversidad de formas que $C$. neoformans puede presentar, se aporta la documentación de formas atípicas observadas en especimenes autopsiados de un hombre de 33 años de edad, donde, además de algunas formas ya informadas, se observaron células con citomorfología no documentadas que pueden confundirse con otras entidades fúngicas. El objeto es facilitar su identificación y remarcar la importancia de la visualización de la cápsula y de la ejecución de propiedades fisiológicas para la correcta identificación del hongo.

\section{MATERIALES Y MÉTODOS}

Muestras estudiadas: trozos de hígado, bazo, riñón y cerebro obtenidos por autopsia de una persona de sexo masculino de 33 años de edad, quien murió sin diagnóstico previo. Los trozos de órganos fueron triturados en morteros estériles para su observación microscópica y cultivos.

El Examen Directo de estos materiales fue realizado en preparados en fresco montados con solución fisiológica y tinta china diluida, y en cortes histológicos coloreados con hematoxilina-eosina.

Cultivos: se practicaron cultivos en medio de agar Sabouraud con cloranfenicol. Se incubaron a $30^{\circ} \mathrm{C}$ y $37^{\circ} \mathrm{C}$ durante 7 días.

Identificación de C. neoformans: fue realizada por estudio de caracteres morfológicos, cultivos y pruebas bioquímicas: producción de ureasa en medio agar urea de Christensen, producción de fenoloxidasa por obtención de pigmento melánico (color café) en agar semilla de girasol, determinación de su capacidad de fermentar azúcares usando agua peptonada, púrpura de bromocresol y glucosa como fuente de carbono.

\section{RESULTADOS}

El examen directo con tinta china y preparaciones coloreadas mostraron la presencia de levaduras capsuladas, la mayoría con tamaño entre límites normales, y otras con diámetro mayor a 10 $\mu$. Las muestras de cerebro presentaron, predominantemente, células brotantes simples acompañadas de pseudomicelios. En hígado, bazo y riñones se observaron abundantes levaduras con cápsulas de grosor variable que, generalmente, duplicaban su diámetro. Las células se presentaron unibrotantes y multibrotantes, con brotes multilaterales y bipolares, cadenas de brotes y pseudomicelios desde rudimentarios a muy desarrollados, dando estructuras caprichosas, muy complejas y formas ramificadas (Figura 1). 

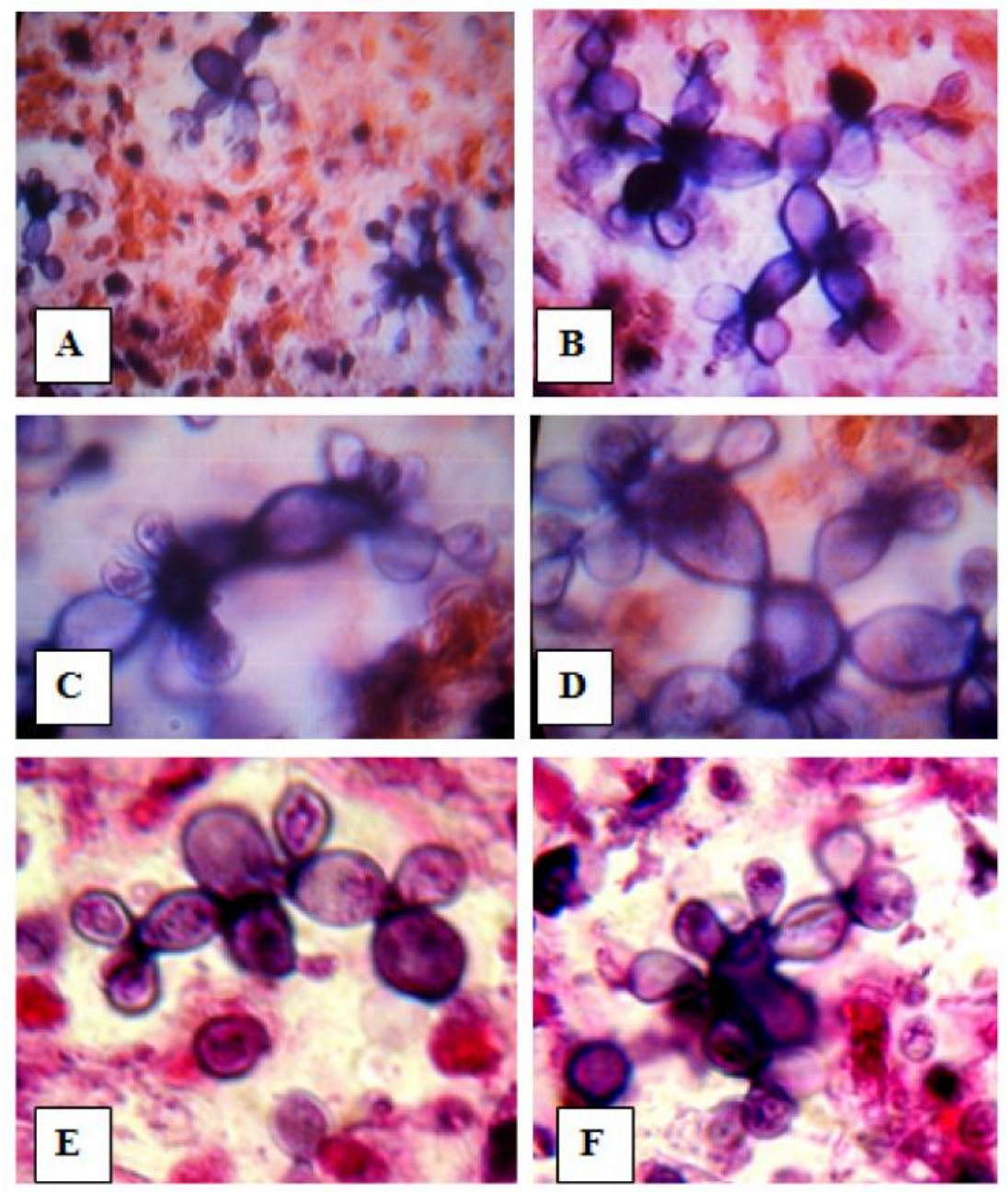

Figura 1: Pseudomicelios muy desarrollados de C. neoformans, formando estructuras muy complejas y formas ramificadas.

Los blastoconidios presentaban forma variada: globosos, claviformes, ovoides a muy alargados. Se observaron blastoconidios unidos por una base ancha a la célula madre. También células dicotómicas sugiriendo ramificaciones ( Figuras $1 \mathrm{E}$ y $1 F$ y $2 A, 2 B, 2 C$ y $2 D)$.

En la mayor parte de los pseudomicelios, las células madres fueron claviformes, 0 con forma de frasco, asentadas sobre su base ancha y brotante por el extremo aguzado dando numerosos blastoconidios que permanecían unidos a las células progenitoras (Figura $1 \mathrm{~A}, 1 \mathrm{~B}, 1 \mathrm{C}$ y $1 \mathrm{D}$ y Figura $2 \mathrm{C}$ y 2D). También, formaron blastoconidios en su base ancha (Figura 2D). En algunas células con brotación multilateral los blastoconidios eran de diferentes tamaños y con distintos grados de desarrollo (Figura 3).

No se observaron conexiones en hebillas entre células de los pseudomicelios. 

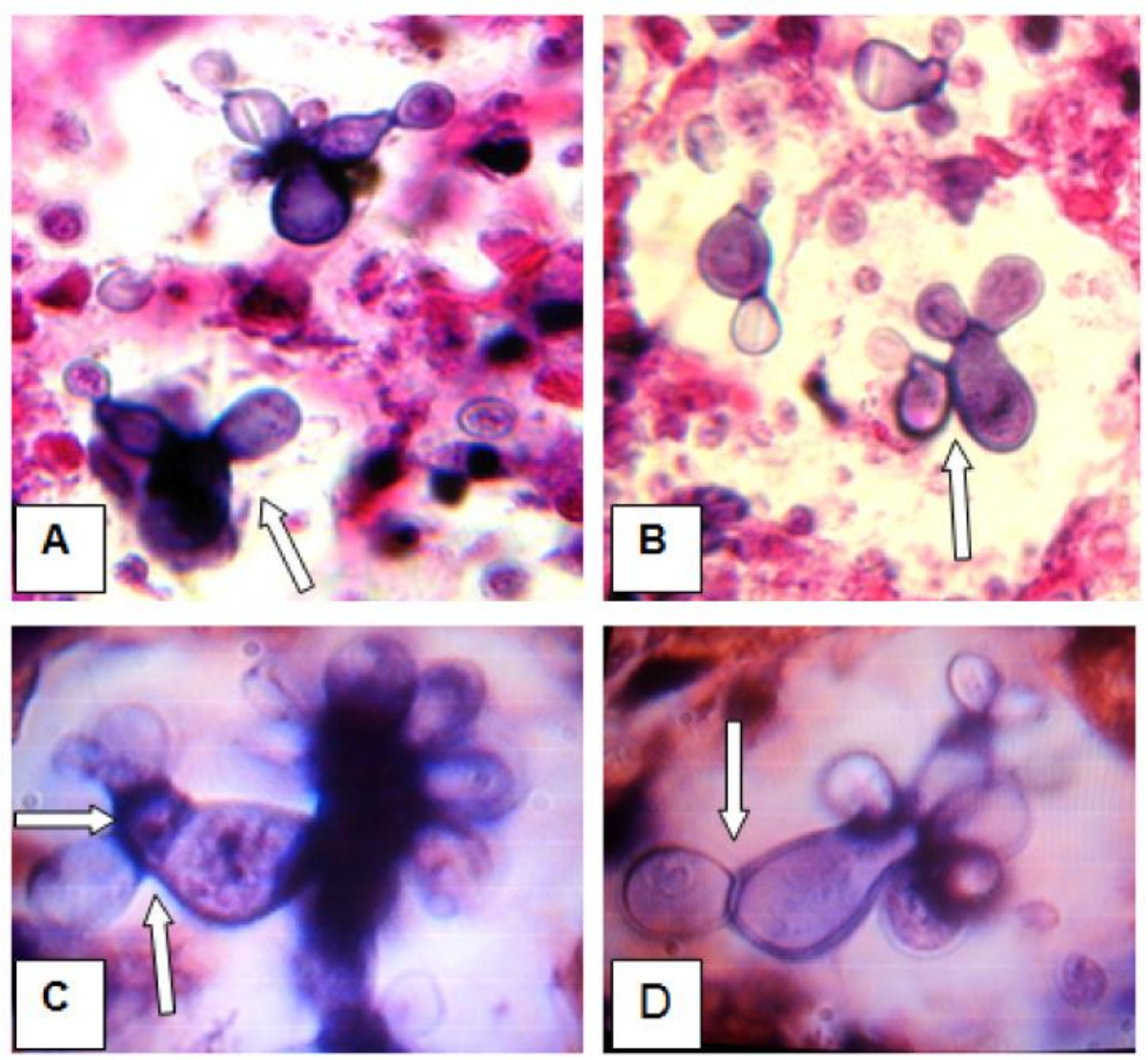

Figura 2: Blastoconidios de C. neoformans de formas variadas y unidos a la célula madre por una base ancha (flechas).

Algunos pseudomicelios mostraron a la célula progenitora con ramificación lateral, solitaria, única, que, a diferencia de las típicas brotaciones de spseudomicelios, no tuvieron lugar en zonas de unión de brotes (Figura 4).

El hongo revirtió a su apariencia normal en cultivos artificiales. Desarrolló dando colonias blancas cremosas que, con el tiempo, fueron crema y del aspecto mucoso característico, constituido por levaduras brotantes, capsuladas, sin pigmentos carotenoides, sin capacidad fermentativa y productoras de ureasa, correspondiendo por estos caracteres, al género Cryptococccus. La especie $C$. neoformans fue identificada por su capacidad para desarrollar a $37^{\circ} \mathrm{C}$, formar fenoloxidasa y pigmento melánico. 
Morfología atípica de Cryptococcus neoformans en tejidos autopsiados - A van Gelderen et al.

\section{DISCUSIÓN}

El aislamiento de $C$. neoformans formó abundantes pseudomicelios de estructuras muy desarrolladas, superando en alto grado, la complejidad de las cepas atípicas documentadas en trabajos previos $(29-31,35,36)$. Algunas recuerdan los pseudomicelios de Cándida patógenas del hombre (con excepción de C. glabrata), a otras levaduras y a algunos hongos filamentosos. Como es sabido, la ausencia de pseudomicelios y de pseudohifas, junto a la formación de blastoconidios unidos a las células madres por una base angosta y la presencia de cápsula, sirven de base para la diferenciación de $C$. neoformans con otros hongos $(1,2,25,29)$. También se observaron levaduras con tamaño mayor que la típica de la especie.

Esta es la primera documentación de $C$. neoformans con blastoconidios unidos a las células progenitoras por una base ancha, brotes distintos a los esféricos 0 elipsoidales y brotes laterales. Células con dicotomía, formas ramificadas raras y formación de blastoconidios que, por su relación con la célula progenitora, recuerda la forma blástica prolífera simpódica de conidiógenesis de hongos filamentosos, donde la célula progenitora tiene algún tipo de crecimiento continuo después de la producción del primer conidio con secuencia simpódica, ya que, aparece un nuevo punto de crecimiento a un lado o abajo del primer conidio (1 y 25). También son atípicos los pseudomicelios con célula apical mayor que la precedente, ya que esto caracteriza a las hifas verdaderas (Figura $4 \mathrm{~A}$ ).

La abundancia de formas atípicas podría sugerir que su formación se relacione con la naturaleza de los tejidos analizados. Sin embargo, también se observaron formas atípicas en otros materiales clínicos como las descriptas por Cruickshank et al. y Paschoal et al. en pus y en líquido
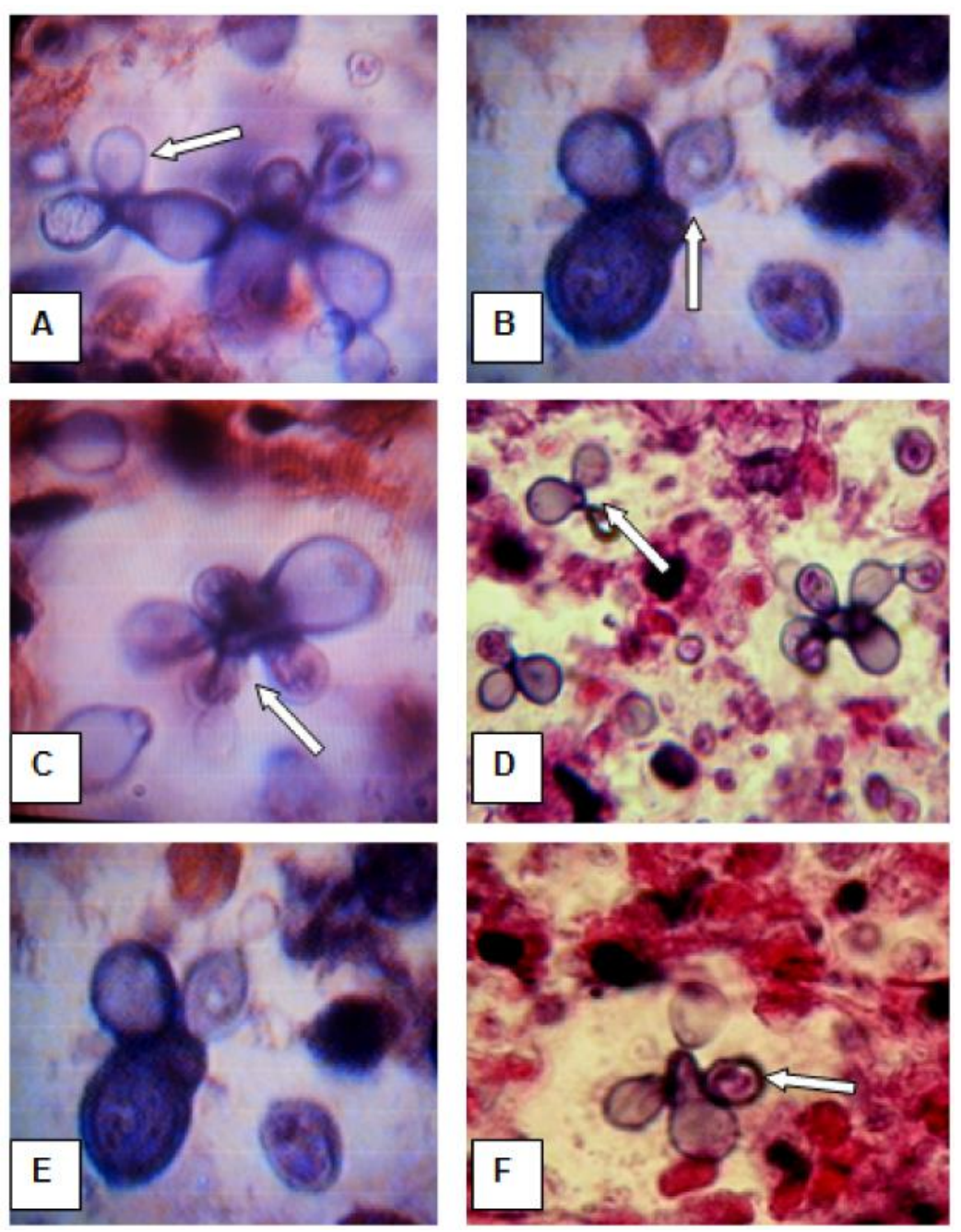

Figura 3. células .multibrotantes de $C$. neoformans con blastoconidios multilaterales de diferentes tamaños, formados a diferentes tiempos.

cefaloraquídeo respectivamente $(36,37)$. De igual manera, podría representar una capacidad de la cepa del hongo.

También fue considerado que la formación de células atípicas y de pseudohifas de $C$. neoformans tiene lugar en pacientes inmucomprometidos, en especial con VIH (37), sin embargo, formaciones similares fueron encontradas en individuos inmunocompetentes $(29,31)$.

De manera similar, se consideró que la formación de pseudohifas indica tendencia de la cepa a formar su teleomorfo $(2,10,29)$. Sin embargo, en este caso, no se observaron conexiones en hebilla que caracterizan al micelio secundario de los Basidiomycetes y que preceden a la formación del teleomorfo. 

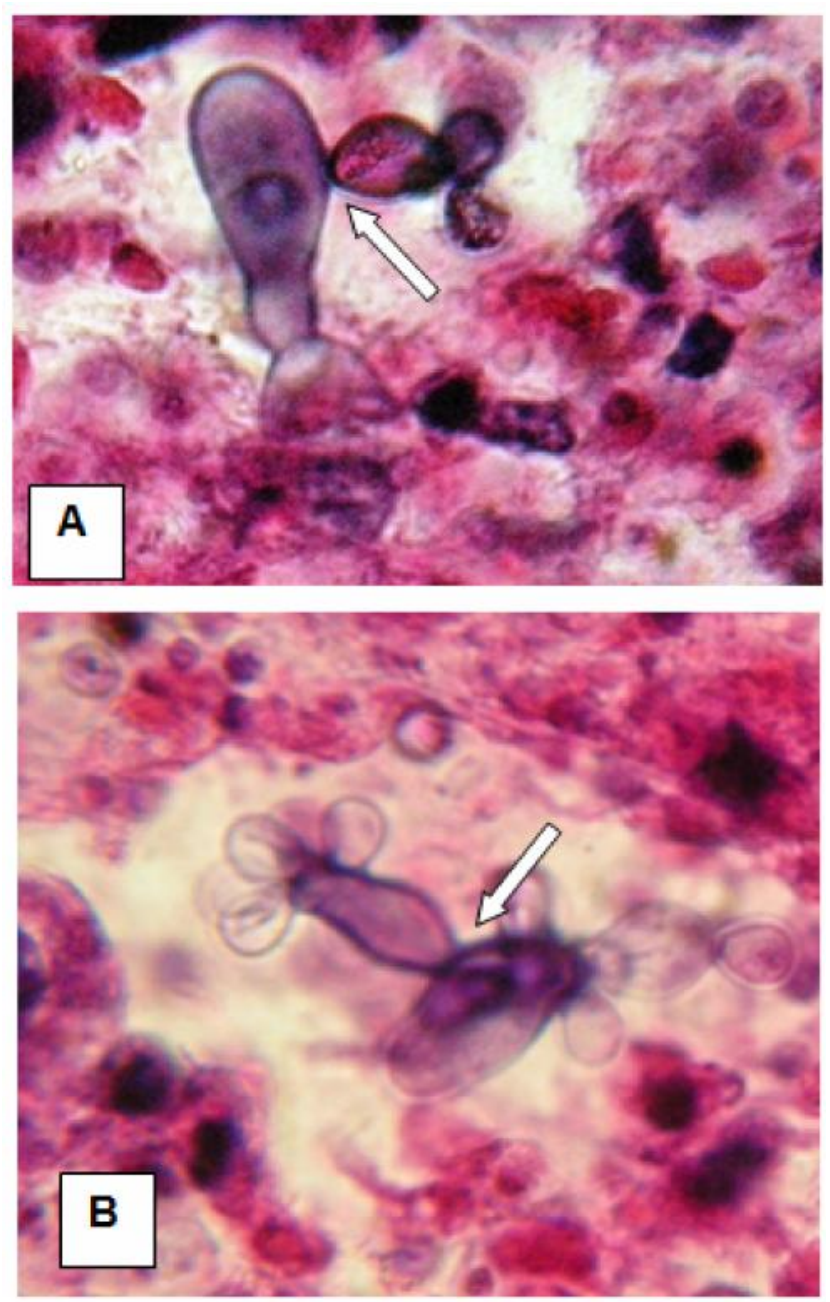

Figura 4: Brotación de células, simple, lateral en pseudomicelios de $C$. neoformans.

Las células con morfología atípica de $C$. neoformans pueden impedir, retardar 0 ser causa de error y confusión con otros hongos durante el diagnóstico de criptococcosis, con graves consecuencias para el paciente. Su reconocimiento se complica en ausencia de otras características útiles para la identificación, como sería la cápsula. Es por esto, que consideramos de gran valor la documentación y difusión de las todas las variantes citomorfológicas de $C$. neoformans que ayuden al pronto reconocimiento de cepas atípicas y orienten la realización de estudios fisiológicos imprescindibles para su identificación.

\section{AGRADECIMIENTO}

Trabajo subsidiado por el Consejo de Investigaciones de la Universidad Nacional de Tucumán, Proyecto 26 D/454 (Directora: Prof. Dra. Aida van Gelderen).

\section{REFERENCIAS}

1. Rippon,J.W. Micología Médica. Hongos y Actinomicetos patógenos. Ed. Interamericana. Mc Graw Hill. $3^{\circ}$ Edición. 1990.

2. Kwon-Chung,K.J. and Bennett,J.E. Medical Mycology. Ed. Lea \& Febiger, Philadelphia, EEUU, 1992.

3. Mitchell,T.G.;Perfect,J.R. (1995). Cryptococcosis in the Era of AIDS-100 Years after the discovery of Criptococcus neoformans. Clin Microbiol Rev 8(4):515-548.

4. Bava,A.J.;Arechavala,A.I.;Robles,A.M.; et al. Criptococosis en nuestro medio (1981-1992). Premio «Alois Bachmann», Academia Nacional de Medicina, 1994

5. Richardon,M.D.\& Warnock,D.W. Fungal Infection. Diagnosis and Management, Blackwell Science Ltd, London. 1997.

6. Bava,A.J.; Robles,A:M.; Negroni,R.; Arechavala,A.; Bianchi,M. (1997). Estudio de algunos aspectos epidemiológicos de 253 casos de criptococosis. Rev Iberoam Micol 14: 111-114.

7. Del Poeta,M.;Casadevall,A.. (2012). Ten Challenges on Cryptococcus and Cryptococcosis. Mycopathologia 173(5-6): 303-310.

8. Franzot,S.P.; Salkin,I.F.; Casadevall,A. (1999).Criptococcus neoformans var. grubii. Separate varietal status for Criptococcus neoformans serotype A isolaties. J Clin Microbiol 37: 838- 840. 
Morfología atípica de Cryptococcus neoformans en tejidos autopsiados - A van Gelderen et al.

9. Casadevall,A.;Perfect,J.R. Cryptococcus neoformans. Washington, DC: ASM press; 1998.

10. Kwon-Chung,K.J. (1976). A new species of Filobasidiella, the sexual state of Cryptococcus neoformans B and C serotipes. Mycologia 68: 942946.

11. Bennett,J.E.;Kwon-Chung,K.J;Theodore,T.S. (1978). Biochemical differences between serotypes of Cryptococcus neoformans. Sabouraudia 16(3):167-174.

12. Kwong-Chung,K.J.;Bennett,J.E. (1984). Epidemiologic differences between the two varieties of C. neoformans. Am J Epidemiology 120:123-130.

13. Speed,B.;Dunt,D. (1995). Clinical and host differences between infections with the two varieties of Cryptococcus neoformans. Clin Infect Dis 21:28.34 .

14. Swinne,D.;De Vroey,C. (1987). Epidemiologie de la Cryptococcose. Rev Iber Micol;4:77-83.

15. Machado,C.C.;Amaral,A.A.;Severo,L.C.. Cryptococcus neoformans var. neoformans isolado do solo. Rev Inst Med Trop Sao Paulo 1993;35:7779.

16. Levitz,S.M. (1991). The ecology of Cryptococcus neoformans and the epidemiology of cryptococcosis. Rev Infect Dis. ;13:1163-1169.

17. Ellis,D.H.;Pfeiffer,T. (1990). Ecology, life cycle and infectious propagule of Cryptococcus neoformans. Lancet;336:923-925.

18. Pfeiffer,T.J.;Ellis,D.H. (1991). Environmental isolation of Cryptococcus neoformans var. gattii from California. J Infect Dis 163:929-930.

19. Pfeiffer,T.J,; Ellis,D.H. (1992). Environmental isolation of Cryptococcus neoformans var. gattii from Eucalyptus tereticornis. J Med Vet Mycol 30:407-408.

20. Pfeiffer,T.; Ellis,D.;. Abstracts of the 13th Congress of the International Society for Human and
Animal Mycology (ISHAM) 1997. North Adelaide, Australia: International Society for Human and Animal Mycology; 1997. Additional Eucalyptus hosts for Cryptococcus neoformans var. gattii, abstr. Flll; p. 106.

21. Lazera,M.S.;Wanke,B.;Nishikawa,M.M. (1993). Isolation of both varieties of Cryptococcus neoformans from saprophytic sources in the city of Rio de Janeiro, Brazil. J Med Vet Mycol 31:449-454.

22. Gezuele,E.; Calegari,L.; Sanabria,D.; Davel,G.; Civila,E. (1993). Isolation in Uruguay of Cryptococcus neoformans var. gattii from anest of the wasp Polybia occidentalis. Rev Iberoam Micol;10:5-6.

23. Ellis,D.H. (1987). Cryptococcus neoformans var. gattii in Australia. J Clin Microbiol. 25:430-431

24. Bava,A.J.;Negroni,R. (1990). Estudio del primer caso autóctono argentino de criptococosis por Cryptococcus neoformans variedad gattii. Rev Iberoam Micol 17: 39-42).

25. De Hogg,G.S.; Guarro,J.; Gené,J.; Figueras,M.J. (2000). Atlas of Clinical Fung ( $\left(2^{\mathrm{a}} \mathrm{ed}\right)$,

26. Feldmesser,M.;Kress,Y.;Casadevall,A. (2001). Dynamic changes in the morphology of Cryptococcus neoformans during murine pulmonary infection. Microbiology 147:2355-2365.

27. Lin,X. (2009). Cryptococcus neoformans: morphogenesis, infection, and evolution. Infect Genet Evol 9:401-416.

28. Zaragoza,0.; Rodrigues,M.L.; De Jesus,M.; Frases,S.; Dadachova,E.; Casadevall,A. (2009). The capsule of the fungal pathogen Cryptococcus neoformans. Adv Appl Microbiol 68:133-216.

29. Williamson,J,D.; Silverman,J.F.; Mallak,C.T.; Christie,J.D. (1996). Atypical cytomorphologic 
appearance of Cryptococcus neoformans: a report of five cases. Acta Cytol 40(2):363-370.

30. Gazzoni,A.F.; Severo,C.B.; Barra,M.B.; Severo,L.C. (2009). Atypical micromorphology and uncommon location of cryptococcosis: a histopathologic study using special histochemical techniques (one case report). Mycopathologia 167:197-202.

31. Gazzoni,A.F.;de Mattos Oliveira,F.;Ferreira $S$ a I I e S, E.; M a y a y O, E .; Guarro,J.;Capilla,J.;Severo,L.C. (2010). Unusual morphologies of Cryptococcus spp. in tissue specimens: report of 10 cases. Rev Inst Med Trop S Paulo 52(3) 145-149.

32. Gazzoni,A.F.;Pegas,K.L.;Severo,L.C. (2008). Histopathological techniques for diagnosing cryptococcosis due to capsuledeficient Cryptococcus: case report. Rev Soc Bras Med Trop 41:76-78.
33. Bottone,E.J.; Toma,M.; Johansson,B.E.; Worserm,G.P. (1986). Poorly encapsulated Cryptococcus neoformans from patients with AIDS. I: Preliminary observations. AIDS Res 2: 211-218.

34. Shadomy,H.J,;Lurie,H.I. Histopathological observations in experimental cryptococcosis caused by a hypha-producing strain of Cryptococcus neoformans (Coward strain) in mice. Sabouraudia 9(1):6-9.

35. Love,G.L.;Boyd,G.D.;Greer,D.L. (1985). Large Cryptococcus neoformans isolated from brain abscess. J Clin Microbiol 22: 1068-1070.

36. Cruickshank,J.G.;Cavill,R.;Jelbert,M. (1973). Cryptococcus neoformans of unusual morphology. Appl Microbiol 25(2): 309-312.

37. Paschoal,R.C.; Melhem,M.S.C.; Guelli,T.; Szeszs,M.W (2007). Atypical Cryptococcus neoformans cells isolated from cerebrospinal fluid samples. Rev Inst Adolfo Lutz 66(1): 78-80. 